M. Serke R. Loddenkemper

\section{Therapeutische Optionen beim malignen} Pleuramesotheliom

\author{
Therapeutic Options in Malignant Pleural Mesothelioma
}

\section{Zusammenfassung}

Beim malignen Pleuramesotheliom stehen mit Operation, Chemo- und Strahlentherapie verschiedene Therapieoptionen zur Verfügung. Bei der Mehrzahl der Patienten stehen Symptomlinderung und Überlebensverlängerung als palliative Therapieziele im Vordergrund. Eine Heilung ist nur in Ausnahmefällen bei Frühfällen durch eine multimodale Therapie mit radikaler OP, Bestrahlung und Chemotherapie zu erreichen. In dieser Übersicht wird die Rolle der Chirurgie diskutiert mit den Verfahren der radikalen extrapleuralen Pleuropneumonektomie oder der palliativen, wenig invasiven Pleurektomie und Dekortikation. Die Rolle der palliativen Bestrahlung mit dem Problem des Verbringens der notwendigen Dosis an die Pleura in enger Nachbarschaft zu Lunge, Herz, Leber und Nieren wird diskutiert. Die Möglichkeiten der Chemotherapie mit „älteren“ Mono- und Kombinationstherapien werden diskutiert. Auch die jüngste große multizentrische Studie, die die Rolle von Cisplatin/Pemetrexed als neuen Therapiestandard beim malignen Pleuramesotheliom begründet, wird dargestellt. Erwähnt werden weitere, experimentelle Verfahren, wie Hyperthermie, Immuntherapie und neue Ansätze der Hemmung von EGFR und VEGF durch „kleine Moleküle“ oder Antikörper. Letztlich muss individuell anhand von Tumorausdehnung, Begleitmorbidität, Allgemeinzustand und Patientenwunsch entschieden werden. Für die Mehrzahl der Patienten kommen thorakoskopische Talkumpleurodese und Chemotherapie, evtl. auch die Pleurektomie und Dekortikation, im Verlauf auch mit Bestrahlung von Problemzonen infrage.

\section{Abstract}

Malignant pleural mesothelioma may be treated with surgery, radiotherapy and chemotherapy. In most patients, the treatment remains palliative with symptom relief and a moderate survival gain. Only a minority of patients with early stage mesothelioma may be cured by a multimodal approach including radical surgery, chemotherapy, and radiotherapy. We discuss the role of surgery with either radical extrapleural pleuropneumonectomy or less invasive palliative pleurectomy and decortication, and the role of radiotherapy, in which the main problem is how to deliver sufficient doses to the pleural surface, sparing radiosensitive structures such as the lung, heart, liver, and kidneys. Chemotherapeutic options are discussed with 'older' mono- and combination regimens and the new promising combination cisplatinum/ pemetrexed, now the 'standard regimen' for malignant pleural mesothelioma. Experimental approaches such as hyperthermia, interferons or interleukins, and 'small molecules' or antibodies inhibiting the EGFR oder VEGF receptor are under clinical evaluation. For the majoritiy of our patients we recommend talcum pleurodesis either by medical thoracoscopy or VATS, followed by chemotherapy with platinum/pemetrexed. Radiotherapy may be applied in case of local tumour growth. The individual therapeutic decision will depend on tumour stage, concomitant diseases, performance status, and on the patient's preference. 
Bildgebende Diagnostik der Pleura [1], Ätiologie, Pathologie und Epidemiologie des malignen Pleuramesothelioms [2] und Diagnostik [3] des Pleuraergusses wurden in den vorhergehenden Folgen der Pleura-Serie dargestellt. Bei einer gegenwärtigen Inzidenz von 1,6 pro 100000 Einwohner [4] muss in Deutschland mit ca. 1000malignen Pleuramesotheliomen pro Jahr gerechnet werden. Bei maximalem Asbestverbrauch in Deutschland zwischen 1965 und 1980 und einer Latenzzeit von ca. 20-40 Jahren ist noch mit einem wesentlichen Anstieg der Inzidenz des MPM in den nächsten Jahren zu rechnen.

\section{Prognose}

Das diffuse maligne Pleuramesotheliom (MPM) ist mit einem medianen Überleben von ca. einem Jahr nach Diagnosestellung in der Prognose dem Bronchialkarzinom vergleichbar [5]. Die EORTC [6] identifizierte anhand Patienten aus Chemotherapiestudien folgende ungünstige Prognosefaktoren: reduzierter Allgemeinzustand, Leukozytose zum Diagnosezeitpunkt, männliches Geschlecht und sarkomatöser Subtyp. Bei operierten Patienten [7] zeigte sich, dass neben der epithelialen Histologie ein Nicht-Befall der mediastinalen Lymphknoten mit einer besseren Prognose einhergeht. Anhand von 155 über 2 Jahre überlebenden Patienten, gesammelt im deutschen Mesotheliom-Register, bestätigte sich der epitheliale Subtyp als günstiger Prognosefaktor, auch waren jüngere $(<60$ Jahre) und weibliche Patienten häufiger unter den 2-Jahres-Überlebenden [8]. Die beobachtete Rate an Langzeit-Überlebenden ( $>2$ Jahre) betrug hier nur 3,5\%. Bei 121 italienischen Patienten [9] betrug das mediane Überleben 10,5 Monate, das 1-Jahresüberleben $40 \%$, das 2-Jahresüberleben $17 \%$ und das 3-Jahresüberleben $8 \%$. Die Multivariat-Analyse ergab einen Vorteil für guten Allgemeinzustand, epitheliale Histologie und aktive Therapie (Pleurektomie und/oder Chemotherapie). Eine englische Studie an 142 Patienten [10] bestätigte die ungünstige Prognose bei nicht-epithelialem Zelltyp, erniedrigtem Hämoglobin-Spiegel, Leukozytose, reduzierten Allgemeinzustand und männlichem Geschlecht. In der Diskussion sind einige biologische Marker, wie z.B. Überexpression des $\alpha$-Folat-Rezeptors [11] oder der Cyclooxygenase-2 (COX-2) [12], welche auch möglicherweise Ziele einer biologischen, „targeted“-Therapie sein könnten.

Klinische Beobachtung ist, dass trotz gesicherter Diagnose eines MPM, unabhängig von der Therapie, einzelne Patienten, z.T. auch ohne Therapie, lange Zeit überleben können.

\section{Key points}

- Prognose malignes Pleuramesotheliom ähnlich dem NSCLC: medianes Überleben ca. 10 Monate,

- 1-Jahresüberleben: 20-40\%, 2-Jahresüberleben: 17\%, 3-Jahresüberleben: $3,5-8 \%$,

- günstige Prognosefaktoren: epitheliale Histologie, guter Allgemeinzustand, fehlende N2-Lymphome.
Klinilk und Diagnostik

Das MPM manifestiert sich als maligner Tumor der Pleura häufig mit Pleuraerguss, begleitet von Luftnot und Thoraxschmerz, später im Verlauf gefolgt von Fatigue und Gewichtsverlust. Es entwickelt sich lange Zeit lokal entlang der Pleura und wächst hier lokal invasiv und infiltrierend mit typischem mantelförmigem Umwachsen der Lunge und Schrumpfung des befallenen Hemithorax. Die lokale Tumorinvasion kann symptomatisch werden mit Schluckstörungen, oberer Einflussstauung, Heiserkeit oder Zwerchfellparese. Bei fortschreitendem Tumor ist häufig im Verlauf ein Durchwachsen des Tumors von der Pleura per continuitatem in das Abdomen zu beobachten, Fernmetastasen entwickeln sich in der Regel spät.

Die Diagnostik kann bei Pleuraerguss schwierig sein, die Thorakoskopie ist hier der sicherste diagnostische Weg, bei fehlendem Erguss können gezielte CT-gesteuerte Stanzbiopsien zur histologischen Diagnose führen (siehe $[1,3]$ ).

\section{Staging}

Problematisch beim MPM ist das Staging, und damit letztlich auch der Vergleich verschiedener Therapieverfahren nach unterschiedlichem Staging. Nachdem früher unterschiedliche StagingSysteme nebeneinander bestanden wird seit 1995 das TNM-System nach UICC [13], basierend auf einer Empfehlung der IMIG (International Mesothelioma Interest Group 1995) am häufigsten zugrunde gelegt. Es setzt eine Beurteilung der lokalen Tumorausbreitung voraus, was mit CT und/oder MRT, optimal invasiv-chirurgisch, zumindest thorakoskopisch gelingt. Ziel war eine genaue Beschreibung der kleinen Gruppe der Patienten mit sehr frühem MPM, die unter Anwendung aggressiver Methoden einen kurativen Therapieansatz haben könnten und eine Definition der Patientengruppen, die zwar lokal ausgedehnter, aber noch resektabel erscheinen. Grundsätzlich bleibt die Stadiierung des MPM schwierig, CT, evtl. MRT sind notwendig, präzise bleibt die IMIG-Stadiierung nur für die Minderheit der chirurgisch stadiierten Patienten.

\section{Key points}

- Klinik: Pleuraerguss, schrumpfender Hemithorax.

- Diagnose per Thorakoskopie oder VATS.

- Stadiierung schwierig, IMIG bester Kompromiss.

\section{Therapieempfehlungen}

Das MPM galt lange Zeit als therapeutisch nicht zu beeinflussende Erkrankung mit entsprechend vorherrschendem therapeutischem Nihilismus.

Die BTS (British Thoracic Society) empfahl noch 2001 [14] beim MPM eine ausschließliche aktive Symptomkontrolle (ASC) mit Betreuung durch einen Spezialisten, adäquate Kontrolle und Therapie physischer, psychologischer und sozialer Probleme. Pleurodese, palliative Bestrahlung und Steroide wurden als adäquate Therapieoptionen empfohlen, Operation oder Chemotherapie wären nur bei individuellem Wunsch und möglichst in Stu- 
dien indiziert. Die ATS [15] (American Thoracic Society) und ATS/ ERS (16) unterscheiden in ihren Empfehlungen zur Therapie des malignen Pleuraergusses 2000/2001 für das MPM nach Stadien: Für das seltene Stadium I mit ausschließlich pleuralem Befall wird eine intrapleurale Interferon/Interleukin-Therapie empfohlen. Für die Stadien II und III wird in Abwesenheit von Studien die Entscheidung zwischen einer multimodalen Therapie mit Talkumpleurodese, Bestrahlung der Eingangsstellen und Kombinations-Chemotherapie oder auch, sofern ein hoch spezialisiertes Thoraxchirurgie-Zentrum zur Verfügung steht, der Einschluss der extrapleuralen Pneumonektomie (EPP) im multimodalen Konzept freigestellt. Für das Stadium IV wurde eine lediglich palliative Therapie empfohlen.

Während noch im Jahr 2002 die Rolle der Chemotherapie beim unresektablen MPM als ungewiss eingeschätzt wurde [17], ist seit 2003 in Kenntnis der Wirksamkeit der neuen Antimetabolite die Einschätzung der Rolle der Chemotherapie deutlich optimistischer, z.T. auch euphorisch [18-21] geworden.

Zurzeit gibt es für das MPM mit der Operation (OP), Chemotherapie und palliativer Bestrahlung etablierte Verfahren, ein therapeutischer Standard unter Wertung der einzelnen Therapiemodalitäten konnte aber bisher nicht etabliert werden.

Spektrum und Radikalität der Therapieansätze reichen von der ultraradikalen trimodal mit Chemotherapie und Bestrahlung ergänzten extrapleuralen Pleuropneumonektomie (EPP) bis zur beobachtenden Symptomkontrolle.

In einem aktuellen, eher nachdenklich wirkenden Editorial 2004 [22] zur Therapie des MPM geben die Autoren zu bedenken, dass zurzeit wahrscheinlich nur kleine Überlebensvorteile zu erreichen sind. Dem Therapeuten wird zum vorsichtigen Umgang mit radikalen Therapieverfahren geraten, um nicht in den ersten symptomarmen Monaten nach der Diagnose durch radikale Therapien mehr zu schaden als zu nutzen.

Gegenwärtig werden die Therapieentscheidungen bei Patienten mit malignem Pleuramesotheliom in der Regel basierend auf klinischer Erfahrung als Einzelfallentscheidungen bei meist palliativem Therapieansatz gefällt. Klinische Studien zum prospektiven Vergleich verschiedener Therapieverfahren sind dringend notwendig, auch wenn es sich um einen bislang relativ seltenen Tumor gehandelt hat.

\section{Key points}

- Therapieempfehlungen bis 2003: kein Standard,

- ab 2003: Cisplatin/Pemetrexed: Standard-Chemotherapie mit ÜL-Vorteil,

- Rolle der Operation (radikale EPP? palliative Pleurektomie?) in der Diskussion.

Fehlende randomisierte Studien, schwierige Stadiierung und Therapiebeurteilung

Bisher gelang es kaum, größere prospektive randomisierte Studien zum Stellenwert einzelner Therapieverfahren durchzuführen. Auch ein Vergleich einer symptomorientiert palliativen
Behandlung („best supportive care“) mit anderen Therapieverfahren wie Operation, Chemotherapie, Bestrahlung, oder der Vergleich von Kombinationen dieser Behandlungen untereinander, fehlen bisher.

Lediglich auf dem Gebiet der Chemotherapie konnte jüngst durch eine randomisierte Studie die Überlegenheit einer 2-fachKombination (Cisplatin/Pemetrexed) gegenüber einer Monotherapie (Cisplatin) gezeigt werden [23].

Erschwert werden Therapiestudien aber auch durch Schwierigkeiten bei der Stadiierung und Beurteilung des Therapieansprechens [24], da sich dieser diffus an der Pleura ausbreitende Tumor, der häufig mit einem Pleuraerguss einhergeht, nach den üblichen RECIST-Kriterien nur schwer erfassen lässt.

\section{Chirurgie}

Zur operativen Therapie stehen zwei Verfahren unterschiedlicher Aggressivität zur Verfügung [25]: einerseits als palliative Maßnahme zur Tumorverkleinerung und Ergusskontrolle die palliative Pleurektomie mit Dekortikation, hier im Folgenden Pleurektomie genannt und andererseits die radikale extrapleurale Pleuropneumonektomie (EPP) in kurativer Absicht.

Problematisch bleibt, dass es aufgrund der Anatomie der Pleura und wegen der Eigenschaft des MPM, früh in benachbarte Strukturen zu infiltrieren, technisch extrem schwer ist, eine mikroskopisch komplette Resektion der gesamten Pleura zu erreichen. Häufig ist technisch nur eine Pleurektomie und Dekortikation technisch möglich, weshalb versucht wurde, die Bestrahlung, z.T. intraoperativ [26], häufig postoperativ und die Chemotherapie ergänzend mit in das Therapiekonzept einzufügen.

Abgeschlossene randomisierte Studien zur Rolle der Operation beim Pleuramesotheliom existieren bisher nicht. Begonnen wurden zwei englische randomisierte Studien: eine VATS-Studie zum Vergleich der Zytoreduktion (Dekortikation) versus alleinige Talkumpleurodese und eine Pilot-Studie (MARS) zum Vergleich der EPP versus Dekortikation im multimodalen Konzept [27].

Man erhofft, mit der Pleuropneumonektomie (EPP) als optimaler Lokaltherapie im multimodalen Therapiekonzept (s.u.), kombiniert mit Strahlen- und Chemotherapie ein längeres Überleben zu erreichen. Ein kurativer Therapieansatz könnte sie bei einzelnen Patienten in sehr gutem Allgemeinzustand und mit guter Prognose (epitheliale Morphologie, kein mediastinaler Lymphknotenbefall) in Betracht kommen.

\section{Extrapleurale Pneumonektomie: EPP}

Bei der extrapleuralen Pneumonektomie (Pleuropneumonektomie: EPP) erfolgt mit dem Ziel der radikalen Tumorresektion (R0-Resektion) eine en-bloc-Resektion der Lunge mit viszeraler und parietaler Pleura, Perikard, ipsilateralem Zwerchfell mit Perikard- und Zwerchfellrekonstruktion.

Morbidität und Mortalität: Erste Ergebnisse der extrapleuralen Pleuropneumonektomie [28] waren mit einer nicht akzeptablen 
OP-Mortalität von bis zu 30\%, erheblicher Morbidität und häufigen Frührezidiven belastet. Mittlerweile konnte die OP-Technik verbessert und die Mortalität an thoraxchirurgischen Zentren auf 3,4-7\% [29-31] verringert werden. Dennoch ist bei $60 \%$ der Operierten mit größeren und kleineren Komplikationen zu rechnen.

Trotz sorgfältiger Patientenselektion gelingt bei der EPP nur bei ca. 70\% der Patienten eine komplette (R0) Tumorentfernung. Japanischen Autoren [30] mit 189 Operationen, davon 116 EPPs, erreichten nur bei $72 \%$ der EPPs eine R0-Resektion. Trotz kompletter Resektion traten bei $43 \%$ der Patienten Rezidive auf. Häufig mussten Zwerchfell, Ösophagus und Perikard mit nicht radikal resezierbaren $(R 1-2)$ Tumorlokalisationen belassen werden. In dieser Patientengruppe unterschied sich das Überleben zwischen den EPP-Patienten und den pleurektomierten Patienten nicht.

Insgesamt deutet die Gesamtzahl der bisher mit EPP behandelten und veröffentlichten Patienten, z.B. die hochspezialisierte Arbeitsgruppe von Sugarbaker aus Boston [29] mit 444 EPP beim Pleuramesotheliom in 23 Jahren, $(=<20$ Pat./Jahr) darauf hin, dass nur für eine Minderheit der Mesotheliom-Patienten dieses Verfahren infrage kommt.

\section{Prognose nach EPP}

Nach EPP ist mit einem medianen Überleben von 9-19 Monaten zu rechnen mit 2-Jahresüberlebensraten von 15-40\%. Kombiniert man die EPP mit adjuvanter Therapie, ist ein 2-Jahresüberleben von 30\%, ein 5-Jahresüberleben von 9\% zu erwarten [29]. Verglichen mit der palliativen Pleurektomie und Dekortikation wurden bei der EPP mit 10\% geringere Rezidivraten als bei der palliativen Pleurektomie mit $52 \%$ beobachtet [31], allerdings wurden nach EPP häufiger Fernmetastasen im abdominellen Raum beobachtet. Nach neoadjuvanter Therapie [32,33] wird nach EPP und Bestrahlung ein vergleichbares 2-Jahresüberleben von $37 \%$ angegeben. Ob sich die neoadjuvante Chemotherapie zu einem „downstaging“ mit besserer Prognose bewährt, bleibt abzuwarten.

Wegen der anfangs hohen Mortalität, der häufig nicht ganz radikalen Operation und der hohen Rate an Lokalrezidiven und Fernmetastasen konnte die EPP keine große Akzeptanz in der Therapie des MPM finden.

Diese Erfahrungen führten zu einer gründlicheren Patientenselektion von Patienten mit günstiger Prognose (kein mediastinaler Lymphknotenbefall, epitheliale Morphologie) mit folgender niedrigerer Morbidität und Mortalität in wenigen erfahrenen Zentren. Die alleinige postoperative Bestrahlung nach EPP senkte das Lokalrezidivrisiko, problematisch war das häufige Auftreten von Metastasen, sodass die Chemotherapie in das multimodale Konzept mit eingeschlossen wurde. Man hofft, durch eine multimodale Therapie mit Kombination von Operation mit Chemound Strahlentherapie (s. u.) Rezidive zu vermeiden und eine Metastasierung zu verhindern (Tab.1).

\section{Palliative Pleurektomie/Dekortikation}

Als chirurgisch-palliative Maßnahmen können die Pleurektomie und Dekortikation zur größtmöglichen Zytoreduktion mit Ver-
Tab. 1 Überleben nach EPP (multimodal) oder Dekortikation

\begin{tabular}{|c|c|c|c|c|c|c|}
\hline $\begin{array}{l}\text { Autor/ } \\
\text { Jahr }\end{array}$ & Therapie & Chemo & $n$ & $\begin{array}{l}\text { MÜZI } \\
\text { Mon }\end{array}$ & 2-J-ÜL & 5-J-ÜL \\
\hline $\begin{array}{l}\text { Rusch } \\
1994\end{array}$ & EPP + Chemo + Rad & CAP & 52 & 16 & $28 \%$ & $18 \%$ \\
\hline $\begin{array}{l}\text { Sugarbaker } \\
1999\end{array}$ & $\begin{array}{l}\text { EPP+/-Chemo } \\
+/ \text { - Rad }\end{array}$ & $\mathrm{Na}$ & 183 & 19 & $38 \%$ & $15 \%$ \\
\hline $\begin{array}{l}\text { Takagi } \\
2001\end{array}$ & $\begin{array}{l}\text { EPP/Pleurect } \\
+/ \text {-Chemo+/-Rad }\end{array}$ & $\mathrm{Na}$ & 189 & 12 & $27 \%$ & $9 \%$ \\
\hline $\begin{array}{l}\text { Rusch } \\
2001\end{array}$ & $\mathrm{EPP}+\mathrm{Rad}$ & Keine & 66 & 17 & $27 \%(3-\mathrm{J})$ & na \\
\hline $\begin{array}{l}\text { Weder } \\
2004\end{array}$ & $\begin{array}{l}\text { Chemo +/-OP } \\
+/ \text {-Rad }\end{array}$ & $\begin{array}{l}\text { DDP/ } \\
\text { Gem }\end{array}$ & 19 & 23 & $37 \%$ & na \\
\hline $\begin{array}{l}\text { Lee } \\
2002\end{array}$ & $\begin{array}{l}\text { Dekortikation + } \\
\text { Rad+/-Chemo }\end{array}$ & CAP & 32 & 18 & $32 \%$ & 12,3 \\
\hline $\begin{array}{l}\text { Serke } \\
2002\end{array}$ & Dekortik + Chemo & $\begin{array}{l}\text { DDP/ } \\
\text { Epi }\end{array}$ & 75 & 15,6 & $\mathrm{Na}$ & $8 \%$ \\
\hline
\end{tabular}

ringerung der Tumormassen und Verhinderung eines Ergussrezidives angewandt werden. Nach Pleurektomie und Dekortikation wird an spezialisierten Zentren eine Mortalität von 1-2\% angegeben [34]. Häufige Komplikationen sind verlängerte Drainagezeiten (ca. 10\%), weiterhin ist mit Pneumonien, Empyemen und Blutungen zu rechnen. Technisch schwierig ist es, die viszerale Pleura vollständig vom Lungenparenchym abzulösen. Hieraus ergeben sich die in der Regel inkompletten Resektionen und die häufigste Rezidivlokalisation ist somit der ipsilaterale Hemithorax [34]. Mit der alleinigen palliativen Pleurektomie und Dekortikation kann keine Heilung, aber eine längerfristig wirksame Kontrolle des Pleuraergusses erreicht werden. Mittlere Überlebenszeiten von 9-20 Monaten nach palliativer Pleurektomie und Dekortikation werden angegeben [35].

In Kombination mit intra- und postoperativer Bestrahlung und adjuvanter Chemotherapie ist mit einem medianen Überleben von 18,1 Monaten und einem 1-Jahresüberleben von $64 \% \mathrm{zu}$ rechnen [26].

Wegen der hohen Rate an Lokalrezidiven entwickelte sich der Trend, an die Dekortikation die adjuvante Bestrahlung [36] oder Chemotherapie anzuschließen.

\section{Key points}

- Die radikale EPP ist in frühen Stadien, bei gutem AZ, epithelialer Morphologie und fehlendem mediastinalen Lymphknotenbefall bei einer Letalität von $3,4-7 \%$ und $25-30 \%$ Morbidität in kurativer Absicht an ausgewählten Patienten (ca. 5-10\% aller Pat. mit MPM) praktikabel.

- Die EPP sollte nur im multimodalen Konzept, möglichst in Studien, mit Strahlen- und Chemotherapie angewandt werden. Sie verspricht ein 2-Jahresüberleben von ca. 30\% und ein 5-Jahresüberleben von ca. 9\%.

- Die Pleurektomie ist ein palliatives Verfahren mit geringer Mortalität und Morbidität zur Ergussprävention und zum „Debulking“ und sollte, möglichst in Studien, mit einer palliativen Chemotherapie kombiniert werden. 
Die Strahlentherapie ist ein wirksames Verfahren beim malignen Pleuramesotheliom, obwohl das MPM nur mäßig strahlensensibel ist. Angesichts der Anatomie der Pleura in enger Nachbarschaft zu strahlensensiblen Organen wie Lunge, Rückenmark, Leber und Niere ist das Verbringen der Strahlendosis an das Zielorgan Pleura eine strahlentherapeutische Herausforderung, naturgemäß leichter praktikabel nach EPP.

Die Bestrahlung beim MPM wird angewandt als

- lokales Verfahren zur Rezidivprophylaxe an den diagnostischen Eingangsstellen,

- als adjuvante Hemithoraxbestrahlung nach EPP oder

- als palliative Hemithoraxbestrahlung.

Eine alleinige kurative Bestrahlung beim Pleuramesotheliom ist bisher aufgrund des ausgedehnten Strahlenfeldes und der Nachbarschaft strahlensensibler Organe nicht gelungen.

In palliativer Absicht ist die Bestrahlung bei der Mehrzahl der Patienten (50-60\%) effektiv zur Schmerzbekämpfung [37,38], z.B. um schmerzhafte Infiltrationen in Rippen, Brustwand, Peritoneum örtlich zurückzudrängen oder auch um ausgedehnte Tumormassen, meist im unteren Hemithorax oder Mediastinum, zu verkleinern.

Das Hauptproblem bleibt, eng benachbarte Strukturen zu schonen. Das Verfahren der Intensitäts-modulierten Strahlentherapie (IMRT) [39] versucht, mittels verschiedener Strahlenblendensysteme (Multileaf-Kollimatoren) die gezielte Applikation der gewünschten Strahlendosis in die Target-Regionen unter Schonung sensibler Organe wie Leber, Niere und Rückenmark zu optimieren.

Die prophylaktische Bestrahlung der Brustwand hat sich nach invasiven diagnostischen Eingriffen in einer randomisierten Studie zur Verhinderung einer Tumoraussaat als wirksam erwiesen. Eine hypofraktionierte Bestrahlung von z.B. 21 Gy über 3 Fraktionen an 40 Patienten konnte das Lokalrezidivrisiko an den Eingangsstellen von $40 \%$ auf $0 \%$ reduzieren [40], wobei empfohlen wird, die Bestrahlung möglichst vor Ablauf von 2 Monaten nach dem Eingriff zu beginnen.

Die Kombination von postoperativer Hemithoraxbestrahlung (40-45 Gy, mit „boosts“ auf Regionen mit hohem Rezidivrisiko) sollte die Lokalrezidivrate senken. Dosislimitierend sind der Spinalkanal (45 Gy), das Herz (45 Gy) und die Lunge (20 Gy). Eine Verschiebung der Abdominalorgane in den unteren Hemithorax nach Pleuropneumonektomie begrenzt die sichere Strahlendosis auf $30 \mathrm{~Gy}$ in den inferioren Arealen [41].

\section{Key points}

- Prophylaktische hypofraktionierte Bestrahlung reduziert Implantationsmetastasen an diagnostischen Eingangsstellen.

- Palliative Bestrahlung verspricht Schmerzlinderung und Verkleinerung von Brustwandmetastasen bei ca. der Hälfte der Patienten.

- Hemithoraxbestrahlung nach EPP gehört zum multimodalen Konzept zur Verbesserung der lokalen Kontrolle.
Die Chemotherapie des malignen Pleuramesothelioms erfolgt unter verschiedenen Intentionen:

- Neoadjuvant/adjuvant/kurativ multimodal bei EPP,

- palliativ adjuvant/neoadjuvant mit Dekortikation,

- palliativ ohne Operation.

Obwohl recht häufig angewandt, hatte die Chemotherapie beim malignen Pleuramesotheliom bisher nur mäßig befriedigende Ergebnisse gezeigt. Abgeschlossene randomisierte Studien, die Chemotherapie mit best supportive care vergleichen, existieren noch nicht.

\section{Monotherapie}

Mehrere Reviews [42 - 44] fassen die Ergebnisse von Studien zusammen, die an kleinen Patientenzahlen lediglich Remissionsraten von unter $20 \%$ beobachteten. Als wirksam mit mäßiger Effektivität und mit Ansprechraten von 10-20\% wurden die Anthrazykline Doxorubicin und Epirubicin, die Alkylantien Mitomycin, Cyclophosphamid und Ifosfamid und die Platinderivate Cis- und Carboplatin eingeschätzt (Tab. 2).

Auch neuere Anthrazyklinanaloga wie Epirubicin, Detorubicin, Pirarubicin und Mitoxanthrone konnten die Ergebnisse nicht verbessern. Ranpirnase (Onconase) als nicht-myelosuppressive Substanz [45], die auf RNA-Ebene wirkt, zeigte, geprüft an 105 Patienten bei geringem Ansprechen ein günstiges 1- und 2-Jahresüberleben (34\% und $21 \%$ ) und wird daher zurzeit randomisiert mit Doxorubicin/Ranpirnase gegen eine DoxorubicinMonotherapie getestet.

Mit Cis- und Carboplatin konnte ein Ansprechen von ca. 15\% [46] erreicht werden. Die Ansprechraten unter Vinorelbine [47] an 29 Patienten betrugen 24\%, das mediane Überleben 13,8 Monate und das 1-Jahresüberleben $41 \%$. Die Kombination Oxaliplatin/ Vinorelbine wurde mit einem Ansprechen von 23\% [48], als nicht besser im Vergleich zu einer Vinorelbine-Monotherapie eingeschätzt. Hochdosistherapien mit Doxorubicin oder Ifosfamid konnten sich beim MPM nicht etablieren [49].

Tab. 2 Monotherapie beim MPM (mod. nach 42)

\begin{tabular}{|llllc}
\hline Substanz & Zahl/Studien & Zahl/Pat. & Response (\%) & $\begin{array}{c}\text { Med. Über- } \\
\text { leben (Mon.) }\end{array}$ \\
\hline Doxorubicin & 2 & 66 & 11 & 7,4 \\
\hline Epirubicin & 2 & 69 & 14 & 10 \\
Cisplatin (DDP) & 2 & 59 & 14 & $5-9$ \\
Carboplatin & 3 & 88 & 11 & $7-9$ \\
Methotrexat & 1 & 60 & 37 & 11 \\
\hline Trimetrexat & 1 & 51 & 12 & $5-9$ \\
Ifosfamid & 3 & 83 & 8 & $8-10$ \\
Vinorelbine & 1 & 57 & 20 & 11,8 \\
Mitomycin C & 1 & 19 & 21 & n.a. \\
Paclitaxel & 1 & 15 & 13 & n.a. \\
\hline
\end{tabular}


Eine Metaanalyse 2002 [17] berücksichtigt 83 prospektive Studien aus den Jahren 1983-2001 mit je mindestens 14 Patienten. Die Therapieschemata wurden in 4 Gruppen verglichen: I: Cisplatinhaltige Therapie, als Mono- oder Kombinationstherapie ohne Doxorubicin, II: doxorubicinhaltige Mono- oder Kombinationstherapien ohne Cisplatin, III: Doxorubicin plus Cisplatin und IV: Mono- oder Kombinationstherapien ohne Cisplatin oder Doxorubicin. Spitzenreiter im Ansprechen war die Kombination Cisplatin/Doxorubicin mit 28,5\%. Cisplatin war die aktivste Mono-Substanz. Der Unterschied im Ansprechen zwischen Cis- und Carboplatin-Kombinationen war mit $24 \%$ vs. $12 \%$ signifikant. Studien mit immunmodulatorischen Substanzen, alleine oder in Kombination, ergaben Ansprechraten von 20\% (Tab.3).

Auch die moderneren Zytostatika der dritten Generation Paclitaxel, Docetaxel, Irinotecan und Gemcitabine (s.u.) wurden beim MPM getestet (Tab.4), wobei Paclitaxel und Docetaxel nur z.T. effektiv zu sein scheinen und die Topoisomerase-Hemmer Irinotecan und Topotecan, obwohl in vitro aktiv an Zelllinien und Capecitabine als oraler Antimetabolit und FU-Abkömmling [50] kein Ansprechen zeigten.

Die EORTC hat in den letzen 15 Jahren sequenziell verschiedene Medikamente (Etoposid, Paclitaxel, Gemcitabine, das Antrazyklin Caelyx, Temozolamid und Tomudex) beim Pleuramesotheliom getestet (siehe Tab.5), wobei lediglich die Daten mit Tomudex Erfolg versprechend schienen und zu einer großen randomisierten Studie führten.

\section{Antifolate}

Methotrexat (MTX) gehört zu den am längsten getesteten Medikamenten beim Pleuramesotheliom. Relativ hoch dosiert, hatte MTX an 60 Patienten ein Ansprechen von 37\% ergeben, allerdings mit einer nebenwirkungsreichen, kostenintensiven und aufwändigen Therapie [51]. Edatrexat, ein MTX-Verwandter, mit und ohne Leukovorin-Rescue [52] getestet, ergab an 58 Patienten ein Ansprechen von 25\%, wobei der Leukovorin-Rescue die Toxizität, aber auch die Effektivität verringerte. Unter moderat dosiertem Trimetrexat [53] ergaben sich Ansprechraten von $12 \%$ und ein medianes Überleben von 7 Monaten.

\section{Neue Antimetabolite}

Gemcitabine, ein Pyrimidin-Antimetabolit, gehört zu den beim MPM wirksamen Substanzen. Eine EORTC-Studie [54] ergab an 27 Patienten nur ein Ansprechen (PR) bei 7\% der Patienten mit einem 1-Jahresüberleben von 33\%. Wesentlich günstiger schätzten Bischoff und Manegold [55] unter Gemcitabine-Monotherapie bei 30 Patienten das Ansprechen von 27\% ein. Eine Arbeitsgruppe aus Chicago [56] sah an 17 Patienten mit eher ungünstig eingeschätzter Prognose kein Ansprechen. Das mediane Überleben betrug nur 4,7 Monate und die Monotherapie mit Gemcitabine wurde hier als unwirksam eingeschätzt.

\section{Gemcitabine + Platinderivat}

Gemcitabine/Cisplatin ergab Remissionsraten zwischen 16 und 48\% [57-59]. Gemcitabine/Carboplatin ergab [60] ein ähnliches Ansprechen (26\%) und medianes Überleben (16,5 Monate). Auch Gemcitabine/Oxaliplatin [61] verspricht ein ähnliches Ansprechen (40\%) und Überleben (13 Mon.) (Tab. 6).
Tab. 3 Meta-Analyse 2002

\begin{tabular}{lcc}
\hline Pat.-Gruppe & Pat.-Zahl & Ansprechraten (\%) \\
\hline $\begin{array}{l}\text { I: Cisplatin: Mono oder Kombi. } \\
\text { (ohne Doxo) }\end{array}$ & 547 & 23,2 \\
II Doxorubicin-Kombin. (ohne Cisplatin) & 213 & 11,3 \\
III Cisplatin/Doxo & 151 & 28,5 \\
IV Mono, andere & 1409 & 11,6 \\
Immunmodulatoren: alleine oder & 432 & 19,9 \\
kombiniert & & \\
\hline
\end{tabular}

Tab. 4 Neuere Substanzen beim mal. Pleuramesotheliom (mod. nach $[18,19])$

\begin{tabular}{|lcc|}
\hline & Pat.-Zahl & Ansprechraten (\%) \\
\hline Docetaxel & 22 & 23 \\
\hline Docetaxel & 20 & 5 \\
Oxaliplatin/Raltitrexed & 58 & 26 \\
\hline Topotecan & 22 & 0 \\
\hline Irinotecan & 28 & 0 \\
Capecitabine & 27 & 4 \\
\hline Vinorelbine & 19 & 21 \\
\hline Ranpirnase & 105 & 0 \\
\hline Vinorelbine/Oxaliplatin & 26 & 23 \\
\hline
\end{tabular}

Tab. 5 EORTC-Studien beim MPM

\begin{tabular}{llcc}
\hline Stud.-Nr & Substanz & Pat.-Zahl & Studie geschlossen \\
\hline 08878 & Etoposid & 10 & 1989 \\
\hline 08924 & Paclitaxel & 14 & 1993 \\
08943 & Gemcitabine & 25 & 1995 \\
08966 & Caelyx & 25 & $9 / 1997$ \\
08976 & Temozolamide & 30 & $10 / 1998$ \\
08992 & Tomudex & 24 & $6 / 2001$ \\
08983 & Tom/DDP vs. DDP & 240 & $1 / 2003$ \\
\hline
\end{tabular}

Raltitrexed (Tomudex ${ }^{\circledR}$ ), ein folat-basierter Thymidilat SynthaseHemmer, ähnlich wie Pemetrexed, war in der Monotherapie bei 35\% wirksam. Die Kombination Raltitrexed/Oxaliplatin ergab, getestet an 70 Patienten, bei 20\% der Patienten eine PR [62]. Ein randomisierter Vergleich zwischen Cisplatin mit und ohne Raltitrexed, von der EORTC an 250 Patienten getestet, ergab ein besseres medianes Überleben, 1-Jahresüberleben und Ansprechen, ohne dass diese Ergebnisse die statistische Signifikanz erreichen konnten [63].

Pemetrexed (Alimta $\left.{ }^{\circledR}\right)$ ist ein neuer Antimetabolit, der 3 Enzyme der Purin- und Pyrimidinsynthese: Thymidilat Synthase: (TS), Dihydrofolat-Reduktase (DHFR) und Glycinamit Ribonukleotidformyl Transferase (GARFT) hemmt, daher auch „Multitarget An- 
Tab. 6 Gemcitabine beim MPM

\begin{tabular}{|lllll}
\hline Medikament & Lit. & Pat.-Zahl & $\begin{array}{l}\text { Ansprech- } \\
\text { raten (\%) }\end{array}$ & $\begin{array}{l}\text { Med. Über- } \\
\text { leben/Mon. }\end{array}$ \\
\hline Gemcitabine & 56 & 17 & 0 & 4,1 \\
Gemcitabine & 54 & 27 & 7 & 8 \\
Gemcitabine & 55 & 16 & 31 & $\mathrm{Na}$ \\
Gem + Cisplatin & 57 & 21 & 47 & 10,3 \\
Gem + Cisplatin & 59 & 25 & 15 & 10 \\
Gem + Cisplatin & 58 & 53 & 33 & 11,2 \\
Gem + Carboplatin & 60 & 50 & 26 & 16,55 \\
Gem + Oxaliplatin & 61 & 25 & 40 & 13 \\
\hline
\end{tabular}

Tab. 7 Antifolate-Mono beim Pleuramesotheliom

\begin{tabular}{llll}
\hline Medikament & Pat.-Zahl & Studien-Zahl & Ansprechen (\%) \\
\hline Trimetrexat & 52 & 1 & 12 \\
Edatrexat & 58 & 1 & 19 \\
Methotrexat & 78 & 3 & 41 \\
Raltitrexed & 17 & 1 & 21 \\
Pemetrexed & 64 & 1 & 14,5 \\
\hline
\end{tabular}

tifolat" genannt. In der Monotherapie mit Pemetrexed [64] wurde an 64 Patienten ein Ansprechen von 14,5\% mit einem medianen Überleben von 10,7 Monaten beobachtet (Tab. 7).

\section{Chemotherapie-Kombinationen}

Polychemotherapien mit herkömmlichen Zytostatika ergaben in Phase-II-Studien ähnliche Remissionsraten wie unter der Monotherapie und eine wirkliche Lebensverlängerung konnte lange Zeit nicht zweifelsfrei bewiesen werden. Symptomlinderungen bei der Mehrzahl der Patienten (62\%) unter einer 3-fach-Kombination (Mitomycin, Vinblastin, Cisplatin: MVP) wurden zwar schon länger beobachtet [65], die aktuelle Metaanalyse [17] belegte jetzt, dass unter Kombinationen das zu erwartende Ansprechen besser als unter einer Monotherapie ist.

Bestätigt wurde dies jetzt durch eine große Phase-III-Studie [23], die im Vergleich Cisplatin gegen eine Cisplatin/PemetrexedKombination einen signifikanten Vorteil zugunsten der Kombination ergab (s.u.).

\section{Randomisierte Studien beim MPM}

Aufgrund geringer Patientenzahlen konnten bisher nur wenige randomisierte Studien unter Einschluss auch nur weniger Patienten beim MPM durchgeführt werden (s. Tab. 8).

Nachdem Pemetrexed beim Mesotheliom Wirksamkeit gezeigt hatte [64] ergab die sog. Emphacis-Studie, randomisiert [23] den Vergleich von Cisplatin gegenüber der Kombination Cisplatin/Pemetrexed mit nun belegter Wirksamkeit im randomisierten Vergleich (s. Tab.9). Diese Studie bestätigte die Überlegenheit der Kombination gegenüber der Monotherapie an der bisher
Tab. 8 Randomisierte Phase-II/III-Studien beim MPM (nach [18])

\begin{tabular}{|lcccl}
\hline & Lit. & $\begin{array}{l}\text { Pat.- } \\
\text { Zahl }\end{array}$ & $\begin{array}{l}\text { Resp. } \\
\%\end{array}$ & $\begin{array}{l}\text { Med. ÜL/ } \\
\text { Mon }\end{array}$ \\
\hline Doxo/CPM vs. Doxo/CPM/DTIC & 66 & 76 & 11 vs. 13 & 7,5 vs. 6,3 \\
\hline DDP/Doxo vs. DDP/Mito & 67 & 70 & 14 vs. 26 & 7,7 \\
\hline DDP/Eto vs. Carbo & 68 & 25 & 8 vs. 0 & 4,7 vs. 5,4 \\
\hline Onconase vs. Doxo & 69 & 154 & Na & 7,7 vs. 8,2 \\
\hline
\end{tabular}

Tab. 9 Cisplatin/Pemetrexed (Alimta ${ }^{\circledR}$ ) versus Cisplatin: an 452 Patienten [23]

\begin{tabular}{|lll}
\hline & $\begin{array}{l}\text { Cisplatin/Pemetre- } \\
\text { xed }\end{array}$ & Cisplatin \\
\hline Ansprechrate & $41,3 \%$ & $16,7 \%$ \\
\hline Med. Überleben/Mon. & 12,1 & 9,3 \\
\hline Zeit bis Progress/Mo & 5,7 & 3,9 \\
\hline Grad III/IV Neutropenie & $27,9 \%$ & $2,3 \%$ \\
\hline Fieberh. Neutropenie & $1,3 \%$ & $0,5 \%$ \\
\hline
\end{tabular}

größten Patientenzahl beim MPM. An 452 Patienten getestet, ergab die Kombination von Cisplatin $\left(75 \mathrm{mg} / \mathrm{m}^{2}\right)$ mit Pemetrexed $\left(500 \mathrm{mg} / \mathrm{m}^{2}\right)$ alle 3 Wochen mit Vitaminsubstitution einen signifikanten Überlebensvorteil von 2,8 Monaten (12,1 Mon. vs. 9,3 Mon.) gegenüber der gleichen Cisplatin-Dosis $\left(75 \mathrm{mg} / \mathrm{m}^{2}\right)$ als Monotherapie. Die Vitaminsubstitution mit Folsäure und Vitamin B12 konnte die anfangs beobachtete Hämatotoxizität deutlich verringern und gehört jetzt zur Standard-Begleittherapie des Pemetrexed. Neben dem signifikant besseren Überleben sah man im Cisplatin/Alimta-Arm eine verbesserte Lungenfunktion, eine längere Zeit bis zur Progression (TTP), eine bessere Ansprechrate ( $41,3 \%$ vs. $16,7 \%$ ) und eine signifikant bessere Lebensqualität.

Eine weitere große, bisher aber nur als ASCO-Abstract veröffentlichte randomisierte Studie mit dem Antifolat Raltitrexed Cisplatin/Raltitrexed (Tomudex) versus Cisplatin [63] ergab zwar einen Vorteil im Ansprechen zugunsten der Tomudex-Kombination, dieser war aber nicht signifikant.

Zusammenfassend ist die Kombination Cisplatin/Pemetrexed die am besten getestete Kombination mit signifikantem Vorteil zugunsten der Kombination. Cisplatin/Pemetrexed ist die derzeitige Standardchemotherapie des MPM.

Eine seit 2000 laufende englische Studie [70], in die 840 Patienten in gutem Allgemeinzustand (WHO Performance Status $0-2$ ) eingeschlossen werden sollen, zeigte bisher, dass ca. die Hälfte der Patienten einer Randomisation zwischen symptomatischer Therapie und einer Chemotherapie zustimmt. Verglichen werden best supportive care mit einer 3-fach Kombination MVP (Mitomycin, Vinblastin und Cisplatin) oder einer Monotherapie mit Vinorelbine. Ergebnisse sind in einigen Jahren zu erwarten. 
Der Versuch, die Chemotherapie als intrakavitäre Chemotherapie direkt an den Tumor im Pleuraspalt zu geben, bewährte sich bisher nicht, da die Penetration in den Tumor offenbar nicht ausreichend war [71]. Die intrakavitäre Therapie wird aber nach wie vor im multimodalen Ansatz nach Pleurektomie getestet, ohne bisher überzeugt zu haben.

\section{Key points}

- Ansprechraten in Monotherapie zwischen 10-20\%.

- Eine große randomisierte Studie zeigt einen ÜL-Vorteil unter der 2-fach Kombination Pemetrexed/Cisplatin gegenüber Cisplatin.

Cisplatin/Pemetrexed zurzeit am besten wirksame Chemotherapie des MPM.

- Gemcitabine-Kombinationen wirksam, bisher nicht randomisiert geprüft.

\section{Zytokintherapie mit und ohne Chemotherapie}

Eine immunologische Genese des MPM wird immer wieder diskutiert, zumal spontane Regressionen, ähnlich wie beim malignen Melanom oder Nierenzellkarzinom beobachtet werden [72] Bei Patienten mit MPM wurden eingeschränkte Immunantworten mit herabgesetzter Mitogen-Antwort oder verringerter Killer-T-Zell-Aktivität gesehen. Es war somit logisch, immuntherapeutische Ansätze, auch in Ermangelung anderer wirksamer Therapieverfahren, beim MPM zu prüfen.

Als relativ gut lokal zugänglicher Tumor, bot es sich an, das MPM intrapleural-intrakavitär und kombiniert mit systemischen Zytokinen, wie Interferon und Interleukin, zu behandeln.

Immuntherapieansätze wie Stimulierung der autologe LAK-Zellen (lymphocin activated killer) durch Interleukine, z.B. durch rekombinantes humanes Interleukin-2 intrapleural, mit und ohne Chemotherapie nach Operation des MPM haben keine überzeugenden Ergebnisse bringen können. Man sah, dass eine derartige Therapie praktikabel ist [73], ohne dass eine eindeutige Wirksamkeit bei nicht zu vernachlässigender Toxizität vorlag. Interleukin-2, über 20 Tage eines 42-tägigen Zyklus subkutan gegeben, ergab bei $8 \%$ der Patienten ein Ansprechen mit einem medianen Überleben von 12 Monaten [74]. Interleukin, intrapleural und subkutan gegeben, ergab bei 31 Patienten [75] ein Ansprechen von $22 \%$ mit einem medianen Überleben von 15 Monaten.

Der postulierte immunmodulatorische und antiproliferative Effekt des Interferon konnte, systemisch gegeben, unter Inkaufnahme deutlicher Toxizitäten nicht belegt werden, so dass dieser Ansatz aufgrund der schlechten Verträglichkeit verlassen werden musste [76]. Im frühen Stadium I wurden unter Interferon- $\gamma$, intrapleural gegeben z.T. lange Überlebenszeiten beobachtet [77]. Letztlich konnte sich die intrapleurale Therapie mit Interleukin $[77,78]$ aber wegen schlechter Verträglichkeit und nur mäßiger Wirksamkeit nicht durchsetzen. Auch wiederholte wöchentliche intrapleurale Infusionen mit aktivierten autologen Makrophagen und $\gamma$-Interferon [79] ergaben nur eine geringe Antitumoraktivität bei schlechter Pleurodese-Wirkung, so dass dieser Therapieansatz nicht weiter verfolgt wird.

Die Kombination von Chemo- und Immuntherapie, Cisplatin, Doxorubicin und $\alpha$-2b-Interferon [80] ergab an 37 Patienten ein recht gutes Ansprechen von 29\%. Das mittlere Überleben von 9,3 Monaten und das 2-Jahresüberleben von 34\% waren aber erkauft mit einer hohen Toxizität im Sinne mit schwerer Myelosuppression und schwerer Fatigue bei je $66 \%$ der Patienten, so dass diese Therapie für viele Patienten zu belastend erscheint. Hochdosiertes Methotrexat ( $3 \mathrm{~g}$ ) mit Leukovorinrescue, 14-tägig gegeben in Kombination mit Interferon ergab ein Ansprechen von 29\% und ein medianes Überleben von 17 Monaten, wobei die Toxizität nicht gering eingeschätzt wurde (81). Die Kombination von intrapleuralem und intradermalem Mycobacterium vaccae (SRLI72) [82] als unspezifischer Immunmodulator mit Chemotherapie (MVP) ergab ein Ansprechen von 37\% und soll randomisiert getestet werden.

\section{Key point}

- Immuntherapien mit Interleukin, Interferon oder anderen Immunstimulantien verbessern theoretisch die Tumorabwehr ohne bisher bewiesene Effektivität beim MPM.

\section{Multimodale Therapie beim MPIM}

\section{EPP im trimodalen Konzept}

In frühen Stadien mit geringer Tumorausdehnung und bei hohem Therapiewunsch der Patienten kommt das Konzept einer multimodalen Therapie infrage. Hier wird die Chemotherapie als neoadjuvante Induktionsbehandlung oder adjuvante Therapie kombiniert mit einem operativen, entweder radikalen (EPP) oder palliativen Verfahren (Dekortikation/Pleurektomie) und anschließender adjuvanter Bestrahlung entweder des Hemithorax (nach EPP) oder der Eingangsstellen (nach Pleurektomie).

Die größte Anzahl mit 183 Patienten mit extrapleuraler Pneumonektomie EPP wertete die Arbeitsgruppe aus Boston über 17 Jahre hinweg im multimodalen Konzept mit adjuvanter Hemithoraxbestrahlung plus Chemotherapie [83] aus. Das mediane Überleben der 176 Patienten, die die Op um mind. 30 Tage überlebten, betrug 19 Monate. Prognostisch besonders günstig erwies sich eine Gruppe von 31 Patienten mit R0-Resektion, epithelialem Zelltyp und ohne mediastinalen Lymphknotenbefall mit einem 5-Jahresüberleben von $45 \%$ nach multimodaler Therapie.

Trotz der aggressiven trimodalen Therapie (EPP) unter Einschluss von Perikard- und Zwerchfellresektionen fanden sich nach einem medianen Intervall von 19 Monaten bei $54 \%$ der Patienten Rezidive, meist im ipsilateralen Hemithorax (35\%) oder im Abdomen (26\%) [31]. Mehr Patienten, als anhand des CT vermutet, hatten intraoperativ einen mediastinalen Lymphknotenbefall (ca. 40\%), so dass eine Optimierung des Stagings (Mediastinoskopie, PET) vor radikaler OP zu prüfen bleibt.

An 57 unterschiedlich operierten Patienten ([54] EPP, 3 Dekortik.) wurde eine Hochdosis-Hemithoraxbestrahlung mit $54 \mathrm{~Gy}$ nach OP getestet [36]. In den Stadien I und II wurde ein günstiges medianes Überleben von 34 Monaten, in den fortgeschrittenen Stadien III und IV ein ungünstiges Überleben von nur 10 Monaten beobachtet. Die überwiegende Zahl von Rezidiven trat als Fernmetastasierung in Erscheinung. Dies kann als Hinweis gewertet werden, dass mit EPP und intensivierter Bestrahlung mit $54 \mathrm{~Gy}$ in frühen Stadien eine ausreichende lokale Kontrolle erfolgt 
war. In den Stadien III und IV ist aber offenbar eine zusätzliche systemische Therapie notwendig.

Ein neoadjuvantes Konzept mit Induktionschemotherapie vor EPP und Bestrahlung könnte, ähnlich wie beim NSCLC im Stadium III, effektiv sein. Nach 3 Zyklen Cisplatin/Gemcitabine mit einem Ansprechen von 32\% wurden in einer Schweizer Pilotstudie [32] von 19 eingeschlossenen Patienten 16 Patienten radikal pleuropneumonektomiert, 13 Patienten konnten nachbestrahlt werden. Das mediane Überleben betrug 23 Monate, das 2-Jahresüberleben $37 \%$. Lediglich 2 der Patienten sind bisher ohne Rezidiv, das rezidivfreie Intervall betrug 16,5 Monate. Bemerkenswert ist, dass nach neoadjuvanter Therapie keine Op-Letalität beobachtet wurde, obwohl die OP von den Chirurgen technisch schwieriger als bei primärer OP beurteilt wurde. Ähnliche Erfahrungen machte eine New Yorker Gruppe [33], die von 19 eingeschlossenen Patienten mit einem Ansprechen von 26\% auf neoadjuvant Cisplatin/Gemcitabine letztlich 9 Patienten mit EPP und adjuvanter Bestrahlung behandelte.

\section{Kombinationen von Dekortikation plus Chemotherapie, Strahlentherapie (intra- und postoperativ), Brachytherapie oder photodynamische Therapie}

Ergänzend zur Dekortikation wurden verschiedene zusätzliche tumorspezifische Maßnahmen erprobt: Die Bestrahlung wurde als Teilkörperbestrahlung palliativ, z.T. mit Brachytherapie ergänzt, adjuvant eingesetzt.

Der Einschluss der Brachytherapie mit Jod-125-Implantation, Iridium-92-Implantation oder postoperativer Phosphor-32-Instillation, kombiniert mit der perkutanen Bestrahlung ergab ein mediane Überleben von 21 Monaten mit einem 2-Jahresüberleben von $40 \%$ und einer Lokalrezidivrate von 30\% [84]. Ein späteres Update der gleichen Patientengruppe ergab nur noch ein medianes Überleben von 13 Monaten, so dass die Brachytherapie keinen Stellenwert in der Therapie des MPM finden konnte.

Die Kombination von Pleurektomie/Dekortikation, intraoperativer und postoperativer Bestrahlung, bei der Hälfte der 26 Patienten zusätzlicher Chemotherapie [26] ergab ein medianes Überleben von 18 Monaten, ein 2-Jahresüberleben von 32\% und in der Regel lokoregionäre Rezidive.

Die photodynamische Therapie mit einem Rotlichtlaser zur Aktivierung zytotoxischer Medikamente im Pleuraspalt nach operativer Tumorverkleinerung („debulking“) ergab in einer randomisierten Studie keinen Vorteil [85] und konnte als zusätzliche lokale Maßnahme nach EPP (7 Pat.) oder Pleurektomie (19 Pat.) [86] das mediane Überleben von 12,4 Monaten und die lokale Kontrolle nicht wesentlich verbessern.

Die Kombination von palliativer Dekortikation/Pleurektomie, gefolgt von intrapleuraler Chemotherapie mit Cisplatin/Mitomycin und anschließender 8-wöchiger Chemotherapie mit Cisplatin/ Mitomycin [87] zeigte, dass nicht alle Patienten (von 28 operierten Patienten wegen Toxizität nur 23) die Chemotherapie erhalten konnten. Es ergab sich ein medianes Überleben von 17 Monaten und ein 2-Jahresüberleben von 40\%. Die häufigsten Rezidivlokalisationen waren Lokalrezidive (16 von 20), so dass davon ausgegangen werden muss, dass das Problem der fehlenden lo- kalen Kontrolle durch die intrapleurale Chemotherapie nicht gelöst werden kann.

Dekortikation, Hemithoraxbestrahlung und Chemotherapie ergaben mediane Überlebenszeiten von 8 Monaten [88] mit einem 2-Jahresüberleben von 20\%. Verschiedene Bestrahlungs- und Chemotherapie-Kombinationen wurden angewandt ohne wesentliche Unterschiede.

Wir beobachteten [89] an 269 konsekutiv behandelten Patienten mit MPM, die entweder nur symptomatisch behandelt wurden, eine Chemotherapie erhielten oder bei denen eine Pleurektomie mit Chemotherapie vorgenommen wurde ein medianes Überleben von 12 Monaten. Die aktiv Behandelten, da nicht randomisiert, sicher auch mit besserem Allgemeinmeinzustand bei der Diagnose, lebten mit alleiniger Pleurektomie 15,5 Monate, mit alleiniger Chemotherapie 13,5 Monate und mit Pleurektomie + Chemotherapie 15,6 Monate, ohne dass diese Unterschiede Signifikanz erreichen konnten.

\section{Vergleich operativer Verfahren: EPP versus Dekortikation mit und ohne Chemotherapie}

Ein systematischer Vergleich zwischen EPP und palliativer Dekortikation erfolgte bisher nicht. Im nicht randomisierten Vergleich seiner Patienten mit EPP oder palliativer Dekortikation fand Takagi [30] keinen Überlebensunterschied: das mediane Überleben betrug in beiden Gruppen 12 Monate, das 2-Jahresüberleben $29,7 \%$ und $26 \%$ und das 5-Jahresüberleben 9,1\% oder 9,5\%. Als Prognosefaktoren wurden in der Multivarianzanalyse Geschlecht und adjuvante Therapie definiert. Die Autoren folgerten daraus, dass die EPP keinen Überlebensvorteil verspräche. Trotz radikaler EPP sei mit einer hohen Rezidivrate zu rechnen.

\section{Pleurektomie mit und ohne Chemotherapie}

An 121 Patienten wurde nicht randomisiert [9] das Überleben in Abhängigkeit von den Therapieoptionen: best supportive care, Pleurektomie alleine, Pleurektomie und Chemotherapie und alleinige Chemotherapie verglichen. Die aktiv Behandelten lebten länger (12 Monate) als die Nicht-Behandelten (4 Monate) und ein Trend deutete einen Vorteil für die Gruppe mit Pleurektomie und adjuvanter Chemotherapie (med. ÜL: 14 Monate) an. Zu berücksichtigen ist hier, dass in dieser nicht randomisierten Kohorte nach Allgemeinzustand, einem wesentlichen Prognosefaktor, entschieden wurde. Manzini [90] fand an 80 nicht randomisierten Patienten beim Vergleich zwischen Operation plus Chemotherapie (15 Monate) oder alleiniger Chemotherapie (13 Monate) keinen signifikanten Überlebensunterschied.

\section{Ganzkörperhyperthermie und Chemotherapie}

Unter der Vorstellung, die Chemotherapie-Wirksamkeit mittels Ganzkörperhyperthermie zu verstärken, wurde die 3-fach-Kombination Carboplatin/Ifosfamid/Etoposid mit einer Ganzkörperhyperthermie kombiniert. An 25 Patienten konnte in einer Phase-II-Studie [91] ein Ansprechen von 20\% gesehen werden mit einem medianen Überleben von 76 Wochen und einem 2-Jahresüberleben von $20 \%$. Gegenwärtig wird dieses Konzept in einer Phase-III-Studie geprüft. Es wurde auch ein positiver Einfluss auf die Lebensqualität beobachtet [92]. 
Morbidität und Mortalität mit niedrigen Rezidivraten bei Ergüssen, die vorher therapieresistent waren [103].

Verschiedene Typen von „Gentherapie“ wurden versucht. Prinzipiell ist das MPM ein attraktives Ziel für eine mögliche Gentherapie, da Standardtherapien kaum definiert sind, der Pleuraraum relativ gut zugänglich ist und im Erkrankungsverlauf für lange Zeit die lokale Tumorausdehnung im Vordergrund steht. Möglich ist ein Einschluss von „viralen Suicide-Genen“, die die Tumorzellen infizieren und dabei empfindlich auf antivirale oder zytotoxische Substanzen machen sollen. Möglich ist auch das Einbringen von immunmodulatorischen Genen, die die Abwehrmechanismen gegen Tumorzellen stimulieren sollen [93]. In einer Phase I Studie wurde versucht, den Tumor mit einem Virus zu infizieren, welches die Zelle veranlasst, unter Therapie mit einem Virustatikum, z.B. Gancyclovir DNA-toxisch und damit zytostatisch zu wirken [94]. Auch Versuche einer Tumorvakzinierung zur Stimulation der Abwehr gegen die Tumorzellen wurden unternommen.

Eine sog. „Antisense-Therapie“ mit Oligonukleotiden, die direkt auf ein apoptotisch wirkendes bcl-xl-Genprodukt wirken sollen, wird an Zelllinien getestet [95].

Zwei Tyrosinkinase-Inhibitoren (TKI) wurden beim MPM getestet und als wirkungslos eingestuft: Imatinib $\left(\right.$ Glivec $^{\circledR}$ ), getestet an 17 Patienten mit MPM war ohne Effekt [96], und auch Erlotinib [97], getestet an 64 Patienten, ergab keine Wirksamkeit beim MPM. Auch Angiogenese-Hemmer werden beim MPM getestet. In klinischen Prüfungen sind zurzeit Tyrosinkinase-Hemmer des VEGF (PTK 787/ZK222584), Thalidomid und der VEGF-Antikörper Bevacizumab [98]. Der Hemmer der VEGF Tyrosinkinase SU5416 wird als antiangiogenetisch wirksame Substanz gegenwärtig beim MPM getestet [99].

\section{Key point}

- Gen-Therapie, EGFR- oder VEGF-Tyrosinkinase-Hemmung und Tumorvakzinierung sind experimentelle Therapieverfahren beim MPM.

\section{Therapie des Pleuraergusses beim MPM}

Die frühe internistische Thorakoskopie (Pleuroskopie) beim vermuteten Pleuramesotheliom sichert die Diagnose durch gezielte Probenentnahme. Eine Pleurodese sollte immer angeschlossen werden, sofern eine palliative Pleurektomie nicht geplant ist. Die thorakoskopische Talkumpleurodese nach vollständiger Ergussdrainage und nach Wiederherstellung des Kontakts zwischen Pleura viszeralis und parietalis ist effizient $[100,101]$ und Therapie der Wahl. Neben der Talkum-induzierten Verschwartung ist auch eine, zumindest in vitro beobachtete direkte Apoptose-induzierende Wirkung des Talkum [102] zu berücksichtigen.

Chirurgische Dekortikation und Pleurektomie sind effektiv in der Ergusskontrolle, sollten aber als invasive Verfahren nur schwierigen Situationen in der Ergusskontrolle (persistierender Erguss, Nichtausdehnen der Lunge, persistierende Fistel) vorbehalten bleiben. Die chirurgische Thorakoskopie (VATS) erlaubt die partielle Pleurektomie im Sinne einer Zytoreduktion mit geringer

\section{Key points}

- Talkumpleurodese ist Therapie der Wahl zur Kontrolle des Pleuraergusses.

Palliative Dekortikation/VATS bei Problem-Ergüssen.

\section{Heckeshorner Therapieempfehlung zum IMPMI}

- Wir empfehlen bei gutem Allgemeinzustand, guter kardiopulmonaler Funktion, epithelialer Morphologie und ausgeschlossenen mediastinalen Lymphomen die Operation, in Einzelfällen die EPP, in der Regel ein „debulking“ mit Pleurektomie und Dekortikation.

- Bei nicht operablem Patienten, bei unseren Patienten die Mehrzahl, empfehlen wir die Talkumpleurodese, gefolgt von 4 Zyklen einer Chemotherapie mit Platin/Pemetrexed.

Bei symptomatischem Tumorprogress sollte die Chemotherapie wieder aufgenommen werden: in Abhängigkeit vom rezidivfreien Intervall wieder mit Platin/Pemetrexed oder, je nach Allgemeinzustand, mit einer Mono- oder Kombinationstherapie unter Einschluss von Gemcitabine, Oxaliplatin, Adriblastin, Vinorelbine oder Mitomycin.

- Die Bestrahlung kann häufig als palliative Hemithoraxbestrahlung zur lokalen Tumorreduktion symptomatisch eingesetzt werden.

- Selbstverständlich ist die symptomorientierte Schmerz- oder Sauerstoffsubstitutionstherapie.

\section{Literatur}

${ }^{1}$ Bittner RC. Bildgebende Diagnostik bei Pleuraerkrankungen. Pneumologie 2004; 58: 238-254

${ }^{2}$ Müller KM. Pleuramesotheliom: Pathologie und Pathogenese. Pneumologie 2004; 58: 670-679

${ }^{3}$ Frank W. Diagnostisches Vorgehen beim Pleuraerguss. Pneumologie 2004; 58: 777-790

${ }^{4}$ Peto J, Decarli A, La Vecchia C et al. The European mesothelioma epidemic. Br J Cancer 1999; 79: 666-672

${ }^{5}$ Astoul P. Pleural mesothelioma. Curr Opin Pulmon Med 1999; 5: $259-268$

${ }^{6}$ Curran D, Sahmoud T, Therasse P et al. Prognostic factors in patients with pleural mesothelioma: the EORTC experience. J Clin Oncol 1998; 16: $145-152$

${ }^{7}$ Sugarbaker DJ, Strauss GM, Lynch TJ et al. Node status has prognostic significance in the multimodality therapy of diffuse malignant mesothelioma. J Clin Oncol 1993; 11: 1172-1178

${ }^{8}$ Neumann V, Rutten A, Scharmach M et al. Factors influencing longterm survival in mesothelioma patients - results of the German mesothelioma register. Int arch occup environ health 2004; 77: 191 - 199

${ }^{9}$ Ceresoli GL, Locati LD, Ferreri AJ et al. Therapeutic outcome according to histologic subtype in 121 patients with malignant pleural mesothelioma. Lung Cancer 2001; 34: 279-287

${ }^{10}$ Edwards JG, Abrams KR, Leverment JN et al. Prognostic factors for malignant mesothelioma in 142 patients: validation of CALGB and EORTC prognostic scoring systems. Thorax 2000; 55: 731-735

${ }^{11}$ Bueno R, Appasani K, Mercer $\mathrm{H}$ et al. The $\alpha$ folate receptor is highly activated in malignant pleural mesothelioma. J Thorac Cardiovasc surg 2001; 121: $1857-1862$

12 Edwards JG, Faux SP, Plummer SM et al. Cyclooxygenase-2 expression is a novel prognostic factor in malignant mesothelioma. Clin Cancer Res 2002; 8: 1857-1862 
${ }^{13}$ Rusch VW. A proposed new international TNM staging system for malignant pleural mesothelioma. From the International Mesothelioma Interest Group. Lung Cancer 1996; 14: 1-12

${ }^{14}$ British Thoracic Society Standards of Care Committee. Statement on the diagnosis and management of mesothelioma. Thorax 2001; 56 : $250-65$

${ }^{15}$ American Thoracic Society. Management of malignant pleural effusions. Am J Resp Crit Care Med 2000; 162: 1987-2001

${ }^{16}$ Antony BVB, Loddenkemper R, Astoul P et al. ERS/ATS Statement: Management of malignant pleural effusions. Eur Respir J 2001; 18: $402-419$

${ }^{17}$ Berghmans T, Paesmans M, Lalami Y et al. Activity of chemotherapy and immunotherapy on malignant mesothelioma. A systematic review of the literature with meta-analysis. Lung Cancer 2002; 38: $111-121$

${ }^{18}$ Tomek S, Emri S, Krejcy K et al. Chemotherapy for malignant pleural mesothelioma: past results and recent developments. Brit J Cancer 2003; 88: 167-174

${ }^{19}$ Tomek S, Manegold C. Chemotherapy for malignant pleural mesothelioma. Past results and recent developnments. Lung Cancer 2004; 45S: $103-119$

${ }^{20}$ Pistolesi M, Rusthoven J. Malignant Pleural mesothelioma: update, current management and newer therapeutic strategies. Chest 2004; 126: $1318-1329$

${ }^{21}$ Eberhardt W. Aufbruch zu neuen Ufern - das Mesotheliom wird eine therapierbare Erkrankung. Pneumologie 2004; 58: 208 - 209

${ }^{22}$ Treasure T, Sedrakyan A. Pleural mesothelioma: little evidence, still time to do trials. Review, Lancet 2004; 364: 1183-1185

${ }^{23}$ Vogelzang NJ, Rusthoven JJ, Symanowski J et al. Phase III study of pemetrexed in combination with cisplatin versus cisplatin alone in patients with malignant pleural mesothelioma. J Clin Oncol 2003; 21: 2636-2644

${ }^{24}$ Klaveren RJ van, Aerts JG, Bruin $\mathrm{H}$ de et al. Inadequacy of the RECIST criteria for response evaluation in patients with malignant pleural mesothelioma. Lung Cancer 2004; 43: 63-69

${ }^{25}$ Ruth S van, Baas P, Zoetmulder FAN. Surgical treatment of malignant pleural mesothelioma: a review. Chest 2003; 123: $551-555$

${ }^{26}$ Lee TT, Everett LL, Shu HK et al. Radical pleurectomy/decortication and intraoperative radiotherapy followed by conformal radiation with or without chemotherapy for malignant mesothelioma. JThorac Cardiovasc Surg 2002; 124: 1183-1189

${ }^{27}$ National Cancer research network trials portfolio. http://www.ncrn.org.uk/portfolio

${ }^{28}$ Butchart EG, Ashcroft T, Barnsley WC et al. Pleuropneumonectomy in the management of diffuse malignant mesothelioma of the pleura. Experience with 29 patients. Thorax 1976; 31 (1): 15-24

${ }^{29}$ Sugarbaker DJ, Jaklitsch MT, Bueno R et al. Prevention, early detection and management of complications after 328 consecutive extrapleural pneumonectomies. J Thorac Cardiovasc Surg 2004; 128: 138 - 146

30 Takagi K, Tsuchiya R, Watanabe Y. Surgical approach to pleural diffuse mesothelioma in Japan. Lung Cancer 2001; 31: 57-65

31 Baldini EH, Recht A, Strauss GM et al. Patterns of failure after trimodality therapy for malignant pleural mesothelioma. Ann Thorac Surg 1997; 63: 334-338

32 Weder W, Kestenholz P, Taverna C et al. Neoadjuvant chemotherapy followed by extrapleural pneumonectomy in malignant pleural mesothelioma. J Clin Oncol 2004; 22: 3451 - 3457

${ }^{33}$ Flores RM, Krug L, Rosenzweig E et al. Induction chemotherapy, extrapleural pneumonectomy (EPP) and adjuvant hemithoracic radiation are feasable and effective for locally advanced malignant pleural mesothelioma (MPM). Proc ASCO, 2004: a 71993

${ }^{34}$ Rusch VW. Pleurectomy/decortication in the setting of multimodality treatment for diffuse malignant pleural mesothelioma. Semin Thorac Cardiovasc Surg 1997; 9: 367-372

${ }^{35}$ Soysal O, Karaoglanoglu N, Demiracan S et al. Pleurectomy/decortication for palliation in malignant mesothelioma: results of surgery. Eur J Cardiothorac Surg 1997; 11: 210-213

${ }^{36}$ Rusch VW, Rosenzweig K, Venkatraman E et al. A phase II trial of surgical resection and adjuvant high-dose hemithoracic radiation for malignant pleural mesothelioma. J Thorac Cardiovasc Surg 2001; 122: $788-795$

${ }^{37}$ Davies SR, Tan L, Ball DL. Radiotherapy in the treatment of malignant mesothelioma of the pleura with special reference to its use in palliation. Austral Radiol 1994; 38: 212 - 214
${ }^{38}$ Bissett D, Macbeth FR, Cram I. The role of palliative radiotherapy in malignant mesothelioma. Clin Oncol 1991; 3: 315-317

${ }^{39}$ Ahamad A, Stevens CW, Smythe WR et al. Intensity-modified radiation therapy: a novel approach to the management of malignant pleural mesothelioma. Int J Radiat Oncol Biol Phys 2003; 55: $768-775$

${ }^{40}$ Boutin C, Rey F, Viallat JR. Prevention of malignant seeding after invasive diagnostic procedures in patients with pleural mesothelioma: a randomised trial of local radiotherapy. Chest 1995; 108: 754-758

${ }^{41}$ Grondin SC, Sugarbaker DJ. Pleuropneumonectomy in the treatment of malignant pleural mesothelioma. Chest 1999; 116: 450S-454S

42 Ong ST, Vogelzang NG. Chemotherapy in malignant pleural mesothelioma. A review. J Clin Oncol 1996; 14: 1007-1017

${ }^{43}$ Ryan CW, Herndon J, Vogelzang NJ. A review of chemotherapy trials for malignant mesothelioma. Chest 1998; 113: 66S-73S

${ }^{44}$ Baas P. Chemotherapy for malignant mesothelioma: from doxorubicin to vinorelbine. Semin Oncol 2002; 29: 62-69

${ }^{45}$ Mikulski SM, Costanzi JJ, Vogelzang NJ et al. Phase II trial of a single weekly intravenous dose of Ranpirnase in patients with unresectable malignant mesothelioma. J Clin Oncol 2001; 20: 274-281

${ }^{46}$ Vogelzang NJ, Goutsou M, Courson JM et al. Carboplatin in malignant mesothelioma: a phase II study of the Cancer and Leukemia Group B. Cancer Chemother Pharmacol 1990; 27: 239-242

${ }^{47}$ Steele JPC, Shamash J, Evans MT et al. Phase II study of Vinorelbine in patients with malignant pleural mesothelioma. J Clin Oncol 2000; 18 : $3912-3917$

${ }^{48}$ Fennell DA, Steele JPC, Shamash J et al. Phase II trial of vinorelbine and oxaliplatin as first-line therapy in malignant pleural mesothelioma. Lung Cancer 2005; 47: 277-281

${ }^{49}$ Talbot SM, Rankin C, Taub R et al. High-Dose ifosfamide with mesna and G-CSF in patients with unresectable malignant mesothelioma. A SWOG study. Cancer 2003; 98: 331 - 336

50 Otterson GA, Herndon II JE, Watson D et al. Capecitabine in malignant mesothelioma: a phase II trial by the Cancer and Leukemia Group B (39807). Lung Cancer 2004; 44: 251-259

51 Solheim OP, Saeter G, Finnanger AM et al. High-dose methotrexate in the treatment of malignant mesothelioma of the pleura. $\mathrm{Br} J$ Cancer 1992; 65: 956-960

52 Kindler HK, Belani CP, Herndon II JE et al. Edatrexate with or without Leukovorin rescue for malignant mesothelioma. Sequential trials by the CALGB. Cancer 1999; 86: 1985-1991

${ }^{53}$ Vogelzang NJ, Weisssman LB, Herndon II JE et al. Trimetrexate in malignant mesothelioma: a cancer and leukaemia group B phase II study. J Clin Oncol 1994; 12: 1436-1442

${ }^{54}$ Meerbeck JP van, Bass P, Debruyne C et al. A phase II study of gemcitabine in patients with malignant pleural mesothelioma; EORTC. Cancer 1999; 85: 2577-2582

${ }^{55}$ Bischoff HG, Manegold C, Knopp M et al. Gemcitabine may reduce tumor load and tumor associated symptoms in malignant pleural mesothelioma. Proc Ann Meet Am Soc Clin Oncol 1998; 17: A 1784

${ }^{56}$ Kindler HL, Millard F, Herndon II JE. Gemcitabine for malignant mesothelioma: A phase II trial by the CALGB. Lung Cancer 2001; 31: $311-317$

${ }^{57}$ Byrne MJ, Davidson JA, Musk AW et al. Cisplatin and gemcitabine treatment for malignant mesothelioma: A phase II study. J Clin Oncol 1999; 17: $25-30$

${ }^{58}$ Nowak AK, Byrne MJ, Williamson R et al. A multicentre phase II study of cisplatin and gemcitabine for malignant mesothelioma. Br J Cancer 2002; 87: 491 - 496

${ }^{59}$ Haarst JMW van, Baas P, Manegold Ch et al. Multicentre phase II study of gemcitabine and cisplatin in malignant pleural mesothelioma. British Journal of Cancer 2002; 86: $342-345$

${ }^{60}$ Favaretto AG, Aversa SM, Paccagnella A et al. Gemcitabine combined with carboplatin in patients with malignant pleural mesothelioma. Cancer 2003; 97: 2791 - 2797

${ }^{61}$ Schütte W, Lauerwald K, Schreiber J et al. Phase-II-Studie zur Behandlung des malignen Pleuramesothelioms mit Gemcitabin und Oxaliplatin. ASCO, 2002: abstr. 1332

62 Fizazi K, Doubre H, Le Chevalier T et al. Combination of raltitrexed and oxaliplatin is an active regimen in malignant mesothelioma: results of a phase II study. J Clin Oncol 2003; 21: 349-354

${ }^{63}$ Meerbeeck JP van, Manegold C, Gaafar R et al. A randomized phase III study of cisplatin with or without raltitrexed in patients with malignant pleural mesothelioma (MPM). An Intergroup study of the EORTC 
Lung Cancer Group and NCIC; Proc. Am Soc Clin Oncol 2004; 22: abst. 7021

${ }^{64}$ Scagliotti GV, Shin DM, Kindler HL et al. Phase II study of pemetrexed with and without folic acid and vitamin B12 as front-line therapy in malignant pleural mesothelioma. J Clin Oncol 2003; 8 (21): $1556-1561$

${ }^{65}$ Middleton GW, Smith IE, O'Brian MER et al. Good symptom relief with palliative MVP (Mitomycin-C, vinblastine and cisplatin) chemotherapy in malignant mesothelioma. Ann Oncol 1998; 9: 269-273

${ }^{66}$ Samson MK, Wasser LP, Borden EC et al. Randomised Comparison of cyclophosphamide, imidazole carcoxamide and adriamycin versus cyclophosphamide and adriamycin in patients with advanced stage malignant mesothelioma: a sarcoma intergroup study. J Clin Oncol 1987; 5: $86-91$

${ }^{67}$ Chahinian AP, Antman K, Goutsou M et al. Randomised phase II trial of cisplatin with mitomycin or doxorubicin for malignant mesothelioma by the Cancer and Leukemia Group B. J Clin Oncol 1993; 11: 1559- 1565

${ }^{68}$ White SC, Anderson H, Jayson GC et al. Randomised phase II study of cisplatin/etoposide versus infusional carboplatin in advanced nonsmall-cell lung cancer and mesothelioma. Ann Oncol 2000; 11: $201-206$

${ }^{69}$ Vogelzang T, Taub R, Shin D et al. Phase III randomized trial of Onconase vs. Doxorubicin in patients with unresectable malignant mesothelioma: analysis of survival. Proc Annu Meet Am Soc Clin Oncol 2000; 18: A 2274

${ }^{70}$ Muers MF, Rudd RM, O'Brien MER et al. BTS randomised feasibility study of active symptom control with or without chemotherapy in malignant pleural mesothelioma: ISRCTN 54469112. Thorax 2004; 59: $144-148$

${ }^{71}$ Markman M, Cleary S, Pfeifle C et al. Cisplatin administered by the intracavitary route as treatment for malignant mesothelioma. Cancer 1986; 58: $18-21$

72 Robinson BWS, Robinson C, Lake RA. Localised spontaneous mesothelioma regression-possible immunological mechanism. Lung Cancer 2001; 32: $197-201$

${ }^{73}$ Astoul P, Picat-Joossen D, Viallat JR et al. Intrapleural administration of interleukin-2 for the treatment of patients with malignant pleural mesothelioma: a phase II study. Cancer 1999; 83: 2099-2104

74 Mulatero CW, Penson RT, Papamichael D et al. A phase II study of combined intravenous and subcutaneous interleukin-2 in malignant pleural mesothelioma. Lung Cancer 2001; 31: 67-72

75 Castageto B, Zai S, Mutti L et al. Palliative and therapeutic activity of IL-2 immunotherapy in unresectable malignant pleural mesothelioma with pleural effusion. Results of a phase II study on 31 consecutive patients. Lung Cancer 2001; 31: $303-310$

${ }^{76}$ Ardizzoni A, Pennucci MC, Castagneto B et al. Recombinant Interferon $\alpha-2 b$ in the treatment of diffuse mal. pleural mesothelioma. Am J Clin Oncol 1994; 17: 80-82

77 Boutin C, Nussbaum E, Monnet I et al. Intrapleural treatment with recombinant $\gamma$-interferon in early stage malignant pleural mesothelioma. Cancer 1994; 74: 2460-2467

78 Astoul P, Viallat JR, Laurent JC et al. Intrapleural recombinant IL-2 in passive Immunotherapy for malignant pleural effusion. Chest 1993; 103: 2009-2013

${ }^{79}$ Monnet I, Breau JJ, Moro D et al. Intrapleural infusion of activated macrophages and $\gamma$-interferon in malignant pleural mesothelioma. A phase II study. Chest 2002; 121 (6): 1921 - 1927

80 Parra HS, Tixi L, Latteri F et al. Combined regimen of cisplatin, doxorubicin and $\alpha-2 b$-interferon in the treatment of advanced malignant pleural mesothelioma. Cancer 2001; 92: 650-656

${ }^{81}$ Halme M, Knuuttila A, Vehmas T et al. High-dose methotrexate in combination with interferons in the treatment of malignant pleural mesothelioma. Brit J Cancer 1999; 80: 1781 - 1785

${ }^{82}$ Mendes R, O'Brien MER, Mitra A et al. Clinical and immunological assessment of mycobacterium vaccae (SRLI72) with chemotherapy in patients with malignant mesothelioma. Brit J Cancer 2002; 86: $336-341$

${ }^{83}$ Sugarbaker DJ, Flores RM, Jaklitsch MT et al. Resection margins, extrapleural nodal status, and cell type determine postoperative longterm survival in trimodality therapy of malignant pleural mesothelioma. Results in 183 patients. J Thorac Cardiovasc Surg 1999; 117: $54-65$
${ }^{84}$ Hilaris BS, Nori D, Kwong E et al. Pleurectomy and intraoperative brachytherapy and postoperative radiation in the treatment of malignant mesothelioma. Int J Radiat Oncol Biol Phys 1984; 10: 325 - 331

85 Pass HI, Temek BK, Kranda K et al. Phase III randomized trial of surgery with or without intraoperative photodynamic therapy and postoperative immunochemo-therapy for malignant mesothelioma. Ann Surg Oncol 1997; 4: 628-633

${ }^{86}$ Friedberg JS, Mick R, Stevenson J et al. A phase I study of Foscan-mediated photodynamic therapy and surgery in patients with mesothelioma. Ann Thorac Surg 2003; 75: 952 - 959

${ }^{87}$ Rusch VW, Saltz L, Venkatraman E et al. A phase II trial of pleurectomy/decortication followed by intrapleural and systemic chemotherapy for malignant pleural mesothelioma. J Clin Oncol 1994; 12: $1156-1163$

${ }^{88}$ Mattson K, Holsti LR, Tammilehto L et al. Multimodality treatment programs for malignant pleural Mesothelioma using high-dose hemithorax irradiation. Int J Radiat Oncol Biol Phys 1992; 24: 643 - 650

${ }^{89}$ Serke M, Schönfeld N, Loddenkemper R et al. Multimodal therapy in malignant pleural mesothelioma (MPM) including surgery and chemotherapy in 269 patients. Eur Resp J 2002; 20 (Suppl. 38): 187s

${ }^{90}$ Manzini VDP, Brollo A, Franceschi S et al. Prognostic factors of malignant mesothelioma of the pleura. Cancer 1993; 72: 410-417

${ }^{91}$ Bakhshandeh A, Bruns I, Traynor A et al. Ifosfamide, carboplatin and etoposide combined with 41.8 whole body hyperthermia for malignant pleural mesothelioma. Lung Cancer 2003; 39: 339-345

92 Bruns I, Kohlmann Th, Wiedemann GJ et al. Bewertung des therapeutischen Gewinns von Patienten mit Pleuramesotheliom unter Therapie mit 41,8 Ganzkörperhyperthermie plus Ifosfamid, Carboplatin und Etoposid (ICE) anhand des modifizierten Brunner-Score (MBS). Pneumologie 2004; 58: 210-216

${ }^{93}$ Albelda SM. Gene therapy for lung cancer and mesothelioma. Chest 1997; 111: $144-149 \mathrm{~S}$

${ }^{94}$ Albelda SM, Wiewrodt R, Sterman DH. Gene therapy for lung neoplasms. Clin Chest Med 2002; 23: 265-277

95 Smythe WR, Mohuiddin I, Ozveran M et al. Antisense therapy for malignant mesothelioma with oligonucleotides targeting the bcl-xl gene product. J Thor Cardiov Surg 2002; 123 (6): 119-198

96 Villano JL, Husain AN, Stadler WM et al. A phase II trial of imatinib mesylate in patients (pts) with malignant mesothelioma (MM) Proc. Ann Meet Am Soc Clin Oncol 2004; 22: abstr. 7200

${ }^{97}$ Garland L, Rankin C, Scott K et al. Molecular correlates of the EGFR signaling pathway in association with SWOG S0218: A phase II study of oral EGFR tyrosine kinase inhibitor OSI-774 (NSC-718781) in patients with malignant pleural mesothelioma (MPM) Proc. Ann Meet Am Soc Clin Oncol 2004; 22: abstr. 3007

98 Vogelzang NJ. Emerging insights into the biology and therapy of malignant mesothelioma. Semin Oncol 2002; 6 (suppl. 18): 35-42

${ }^{99}$ Kindler HL, Vogelzang NJ, Chien K et al. SU5416 in malignant mesothelioma: a Universitiy of Chicago Phase II Consortium Study. Proc Ann Meet Am Soc Clin Oncol 2001; 19: abstr. 1359

${ }^{100}$ Loddenkemper R. Management der malignen Pleuraergüsse. Pneumologie 2005; 59: 120-135

${ }^{101}$ Kennedy L, Sahn SA. Talc pleurodesis for the treatment of pneumothorax and pleural effusion. Chest 1994; 106: 1215-1222

102 Nasreen N, Mohammed KA, Dowling PA et al. Talc induces apoptosis in human malignant mesothelioma cells in vitro. Am J Resp Crit Care Med 2000; 161: 595-600

${ }^{103}$ Waller DA, Morritta GN, Forty J. Video-assisted thoracoscopic pleurectomy in the management of malignant pleural effusion. Chest 1995; 107: $1454-1456$

\section{Bereits publizierte Beiträge zu dieser Serie:}

${ }^{1}$ Bildgebende Diagnostik bei Pleuraerkrankungen. Pneumologie 2004; 58: $238-254$

2 Pleura: Pathologie nicht-neoplastischer Erkrankungen. Pneumologie 2004; 58: 516-524

${ }^{3}$ Pleuramesotheliom - Pathologie und Pathogenese. Pneumologie 2004; 58: 670-679

${ }^{4}$ Diagnostisches Vorgehen beim Pleuraerguss. Pneumologie 2004; 58 : $777-790$

${ }^{5}$ Management der malignen Pleuraergüsse. Pneumologie 2005; 59: 120-135 